Ann. Zootech., I975, 24 (3), 595-602.

\title{
APPAREILS DE COLLECTE TOTALE DES FÈCES POUR AGNEAUX EN ALLAITEMENT OU A L'ENGRAIS ET POUR BREBIS
}

\author{
M. TISSIER, G. BECHET et G. MOLÉNAT \\ avec la collaboration technique de P. DacheuX et M. MAQUere \\ Station de Recherches sur l'Élevage des Ruminants, \\ Centre de Recherches de Clermont-Ferrand, I. N. R. A., \\ Theix, Saint Genès Champanelle, 63110 Beaumont
}

\section{RÉSUMÉ}

Les équipements décrits présentent tous comme principale particularité d'être constitués de récipients en plastique moulé rigide qui ne s'écrasent pas et conservent leur forme.

Pour les agneaux en allaitement, on utilise un bécher de $150 \mathrm{ml}$ enfermé dans une enveloppe en forte toile qui joue le rôle de collecteur et vient s'appliquer autour de l'anus de l'animal. L'originalité de l'appareil réside dans l'absence de harnais remplacé par des pièces de toile de jute collées directement sur la toison.

Le sac pour les agneaux à l'engrais est conçu de la même façon que pour les agneaux en allaitement, mais il est plus volumineux et le bécher est remplacé par un seau plastique de 2,5 litres. Un harnais, réalisé en cuir, est nécessaire pour cet équipement.

Pour les brebis, un bidon plastique de 5 litres soutenu par un harnais fait office de sac à fèces. Des perforations assurent le drainage de l'urine.

La collecte totale des fèces est nécessaire à beaucoup d'études nutritionnelles, particulièrement pour connaître l'utilisation digestive des composants d'un régime, ou pour estimer la quantité d'herbe consommée par des animaux au pâturage.

Différents auteurs ont décrit des appareils de collecte pour des agneaux pendant la phase d'allaitement (Brisson et al., r970) ou des agneaux et des brebis au pâturage (Royal, I968; ARNOLD, I960). Ces appareils doivent être robustes et assurer une collecte et un échantillonnage simples et rapides des fèces, sans pertes, en particulier lorsque les moutons sont couchés. La gêne qu'ils causent à l'animal doit être minimale de façon à éviter d'éventuelles blessures ou irritations cutanées, voire des troubles du comportement et des réactions d'affolement. Les appareils décrits ne satisfont généralement pas à toutes ces conditions simultanément. C'est pourquoi nous avons accordé une attention particulière à la confection de matériels adaptés à la conformation des animaux et propres à éviter les inconvénients précités. L'utilisation de récipients en plastique 
moulé rigide (bécher, seau ou bidon) au lieu de sacs en nylon ou en tissu permet de conserver la consistance initiale des fèces, ce qui facilite les manipulations et l'échantillonnage, et évite leur altération éventuelle.

\section{ÉQUIPEMENT POUR AGNEAUX MÂLES EN ALLAITEMENT}

Le sac est constitué d'une cnveloppe en forte toile renfermant un bécher de $150 \mathrm{ml}$ destiné à recueillir les fèces (fig. I). L'ensemble pèse environ $100 \mathrm{~g}$.

Il est attaché par 4 pièces en toile de jute collées directement sur la laine de l'agneau ( $\left.{ }^{1}\right)$ et portant chacune une boucle où viennent s'accrocher 4 sangles solidaires du sac (2 sangles sur le dos, et 2 autres entre les pattes arrière).
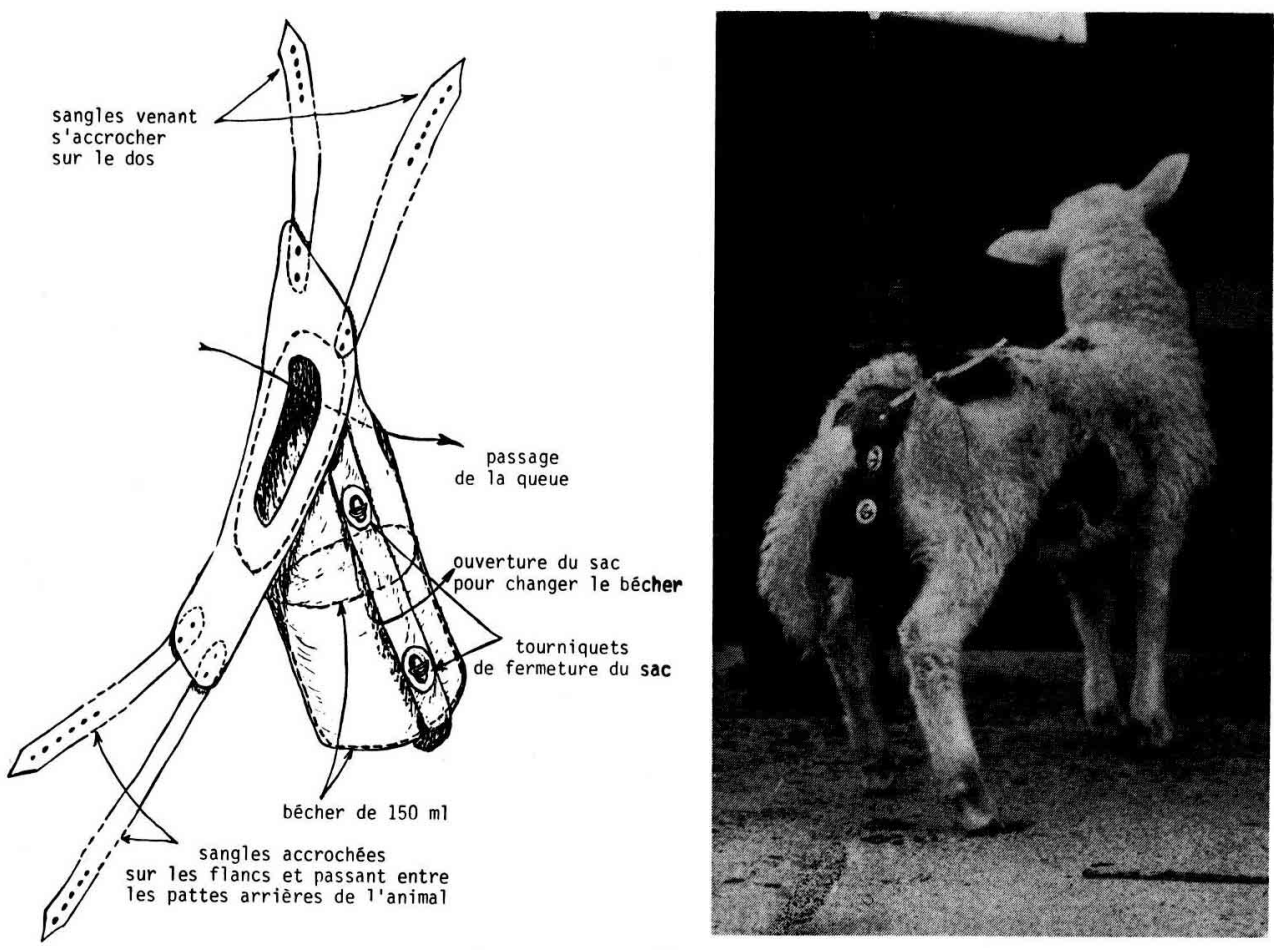

JiG. I. - Liquipement pouragneaux à l'allaitement

Schéma du sac et vue d'un agneau équipé

Cet appareil peut être mis en place sans difficultés grâce aux sangles et aux boucles ì pointe qui permettent un réglage précis. II ne cause qu'une gênc minime ct ne perturbe donc pas le comportement des animaux. L'autre avantage de l'absence de harnais réside dans le fait que les agneaux, bien qu'étant en croissance, peuvent être utilisés tout au long de la phase d'allaitement (de la naissance à l'âge de 2 mois, poids vif compris entre 3 ct I $8 \mathrm{~kg}$ cnviron) sans équipement supplémentaire, car les pièces de toile collées sur lo dos et sur les flancs restent bien en place.

(1) Nous avons utilisé des colles spéciales pour textiles, indifféremment les marques "Tiss-Colle " et "Texticroche". 
L'utilisation de béchers résistants à la chaleur et dont la tare a été préalablement rclevéc permet une grande rapidité des différentes opérations. Il suffit en effet de prélever chaque jour le bécher plein grâce à l'ouverture ménagée sur le côté de l'enveloppe, et de le remplacer. On peut ensuite, après l'avoir pesé, le mettre directement à l'étuve à roo ${ }^{\circ} \mathrm{C}$ pour la détermination de la quantité de matière sèche excrétée.

\section{ÉQUIPEMENT POUR AGNEAUX MÂLES A I'ENGRAIS}
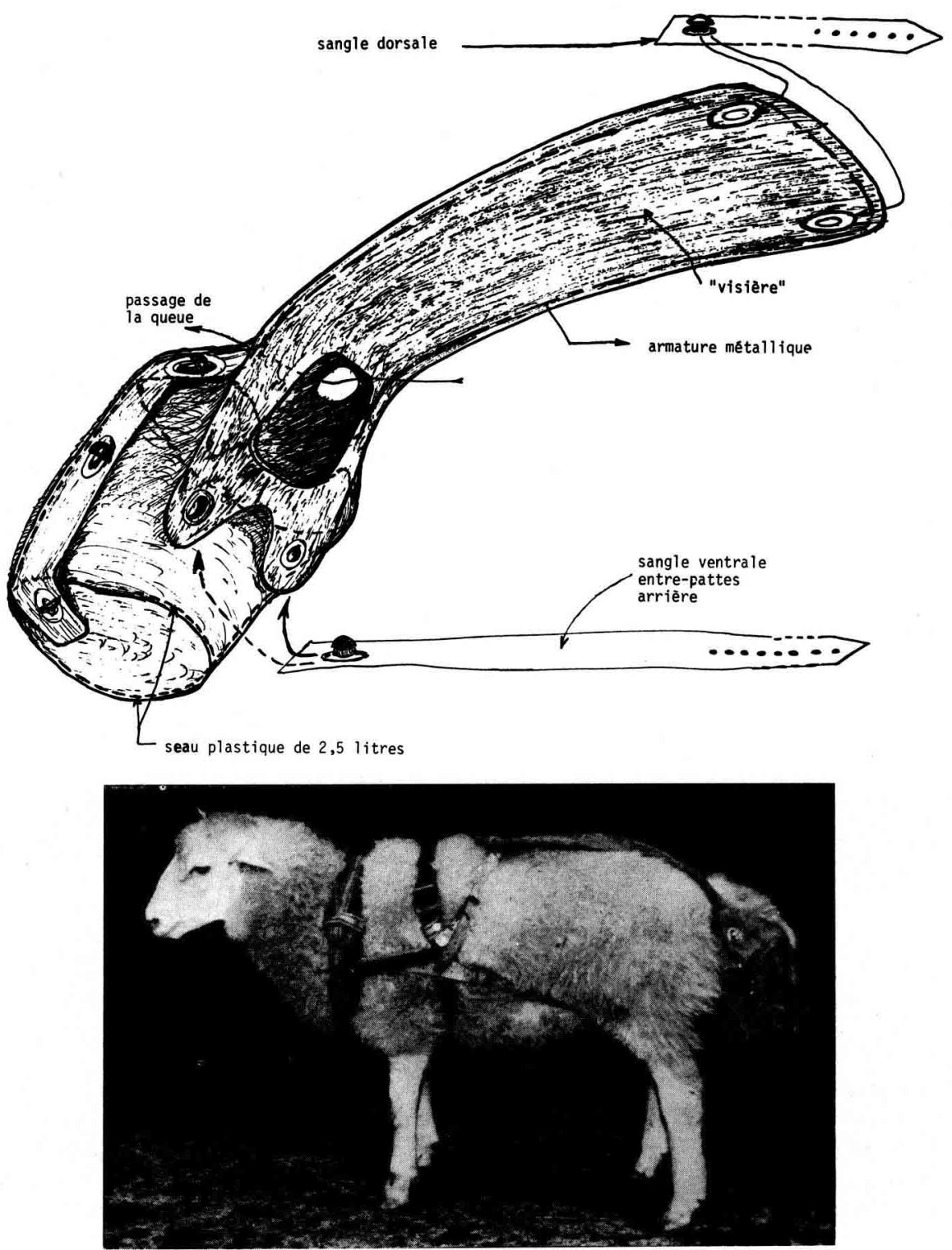

FIG. 2. - Equipement pour agneaux à l'engrais Schéma du sac et vue d'un agneau équipé 
La conception de ce sac (fig. 2) est analogue à celle des agneaux en allaitement. Le bécher est remplacé par un seau plastique de 2,5 litres adapté au volume des fèces à recueillir pour des agneaux jusqu'à $40 \mathrm{~kg}$ de poids vif (âge variant entre 2 et 5 mois environ).

Le poids plus important des fèces (parfois plus de I kg) nous a conduit à prolonger le sac en avant par une sorte de "visière " en tissu ave des armaturas métalliques. Cette visière vient s'accrocher sur un harnais, grâce à des sangles dorsales. Le poids est ainsi répatri sur le dos de l'animal et le sac reste mieux en place, même lorsqu'il est plein.

La figure 3 montre le harnais rendu nécessaire par le poids plus important des fèces. Il est confectionné en cuir et sa découpe a été spécialement étudiée pour s'ajuster au mieux sur l'animal,

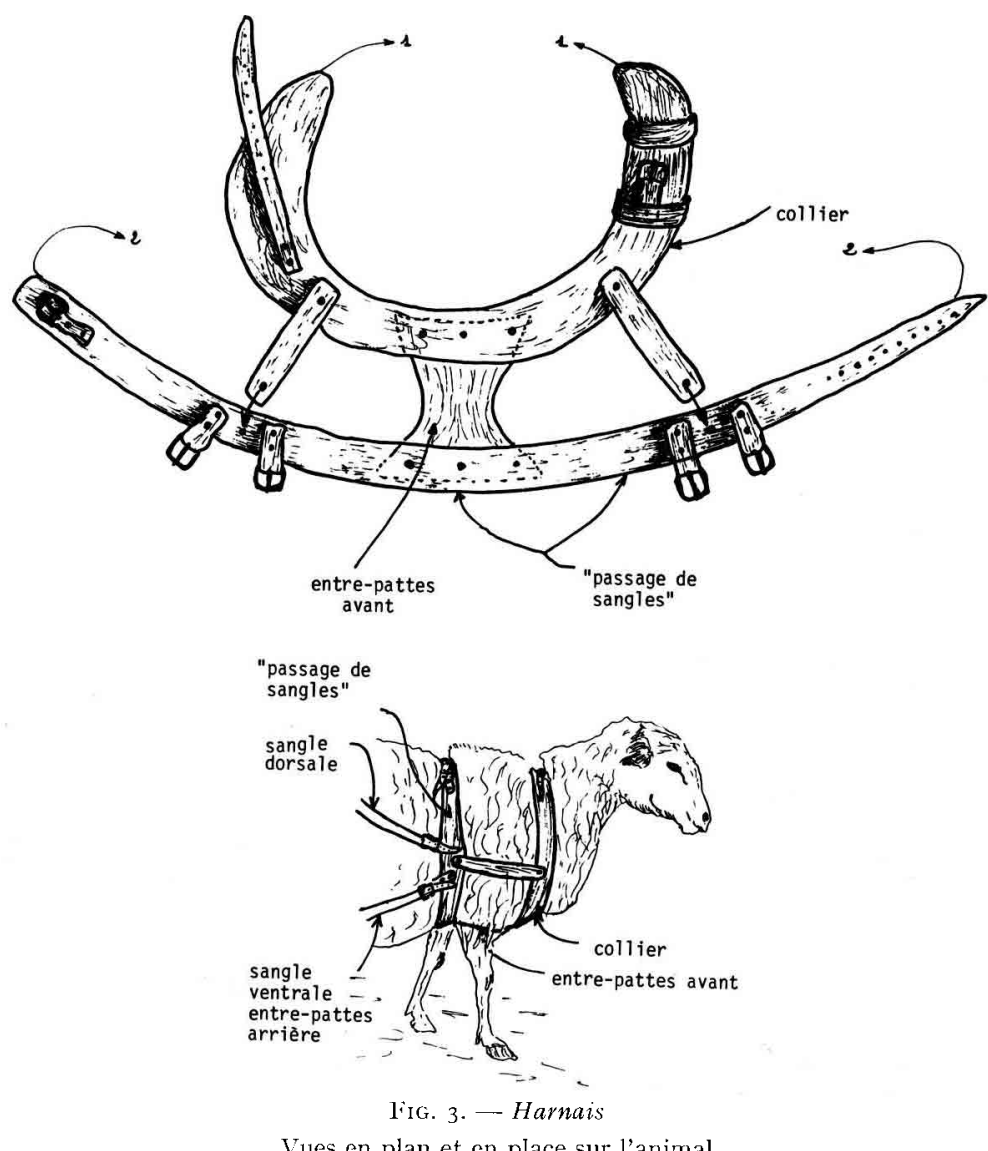

Vues en plan et en place sur l'animal

particulièrement en avant des épaules. La partie antérieure de ce harnais constitue donc un collier ; la partie postérieure se place immédiatement en arrière de l'épaule et des pattes antérieures (à cléfaut de cuir, cette dernière peut être fabriquée avec de fortes sangles en tissu). Elle est munie de boucles à pointes qui permettent d'accrocher les 4 sangles du sac (fig. 2.). Les dimensions de ce harnais doivent être adaptées suivant le format des animaux auxquels il est destiné.

Le port de ce harnais, même pendant une longue période, ne blesse pas les animaux, et ne leur occasionne pas de gêne importante puisqu'il reste bien en place, "sans être fortement serré. 


\section{ÉQUIPEMENT POUR BREBIS AU PÂTURAGE:}

La liberté et la possibilité de courir laissées aux brebis dans les pâtures, imposent à cet équipement une robustesse particulière.

Lc harnais est le même que celui des agneaux à l'engraissement, en adaptant les dimensions.

Le sacs à fèces est constitué d'un bidon en polyéthylène souple de 5 litres et d'un collecteur (fig. 4) venant s'appliquer sur les orifices ano-génito-urinaires de la femelle. Des perforations dans la partic inférieure du bidon assurent le drainage de l'urine.

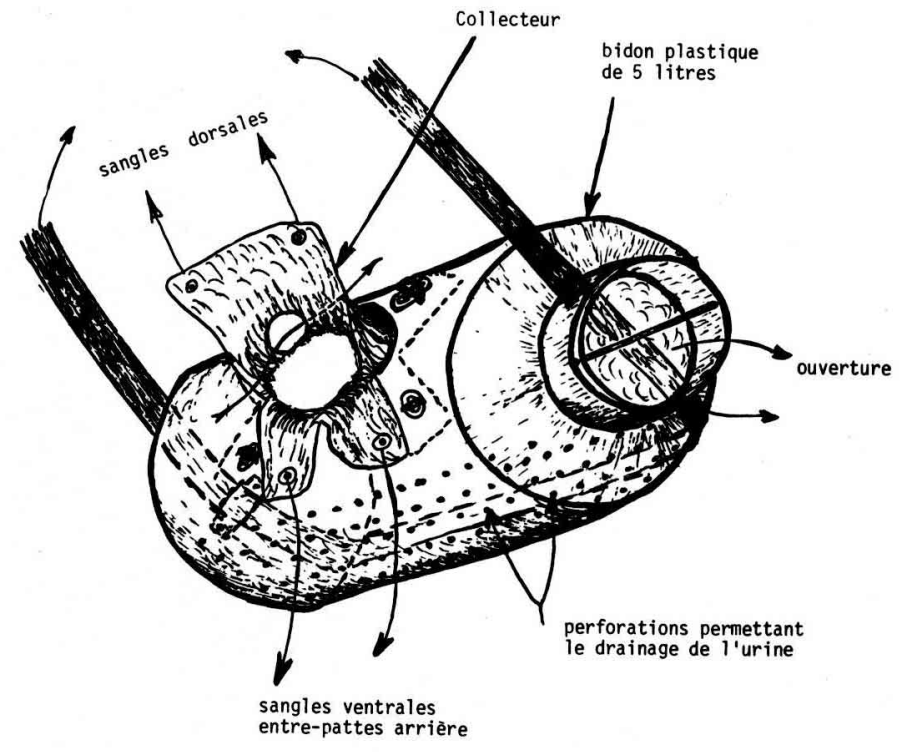

FIG. 4. - Ensemble " bidon + collecleur" pour brebis

L'ensemble bidon + collecteur est maintenu sur l'animal grâce à des sangles qui viennent s'accrocher sur le harnais (fig. 5).

Le couvercle amovible du bidon plastique permet la vidange latérale des fèces sans démonter l'équipement qui peut être utilisé sans inconvénient pendant l'allaitement (fig. 5).

\section{UTIIISATION DE CES ÉQUIPEMENTS E'T PERSPECTIVES}

Les équipements décrits ont été utilisés lors de différentes expérimentations et nous présentons, à titre d'exemple, quelques résultats obtenus.

Les coefficients d'utilisation digestive observés de quatre laits de remplacement d'une part 
(tabl. I) et d'un régime d'engraissement riche en concentré d'autre part (tabl. 2) sont en parfaite concordance avec des résultats obtenus par ailleurs avec des aliments comparables mais par d'autres méthodes ou d'autres types d'appareils (Andrews, KaY et Orskov, 1969 ; Molénat et Theriez, I974; Tileriez, Protais et Molénat, I974). Les faibles écarts-types observés montrent 'que l'cmploi de ces équipements n'entraîne pas de perturbation importante des animaux.
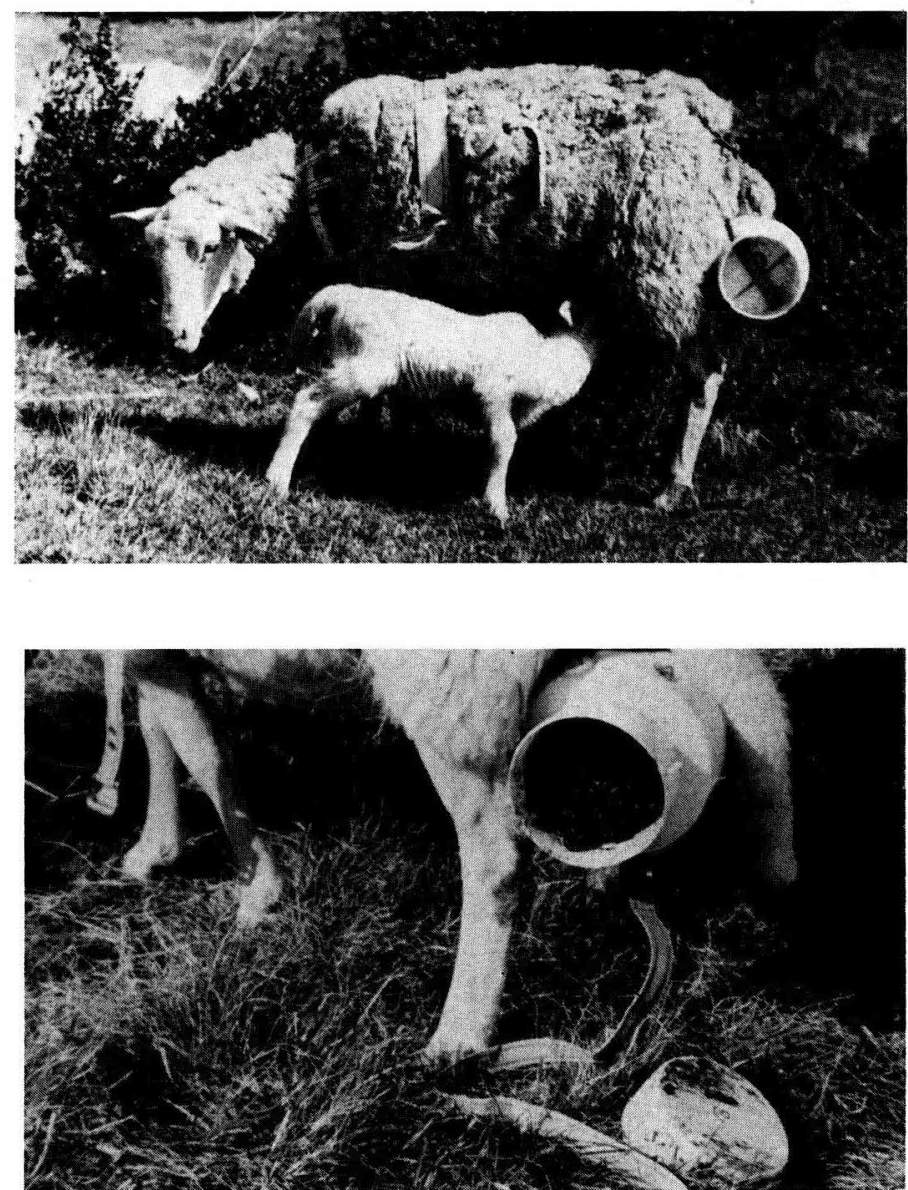

FiG. 5. - Equipement pour brebis

Le "sac " en place sur une femelle allaitante et sa.vidange par simple ouverture du couvercle

L'appareil pour brebis au pâturage n'a pas été utilisé pour effectuer des mesures ${ }^{-}$de digestibilité avec des femelles. Il serait nécessaire en effet, de mesurer auparavant les variations introduites (en particulier pour les matières azotées) par la percolation de l'urine au travers de la masse des fèces. En revanche, cette percolation n'entraîne vraisemblablement pas de perte sensible de matière sèche. La précision de la mesure de la quantité de matière sèche fécale est donc satisfaisante et peut permettre d'estimer la quantité ingérée par un animal (tabl. 3) lorsque la digestibilité du régime est connue par ailleurs.

Ces appareils, qui n'imposent aux animaux qu'une contrainte légère, devraient permettre 


\section{TABLEAU I}

Agneaux en allaitement

Comparaison de la digestibilité de + laits de remplacement

\begin{tabular}{|c|c|c|c|c|}
\hline \multicolumn{2}{|c|}{ : } & \multirow[b]{2}{*}{$\begin{array}{l}\text { Nombre } \\
\text { d'agneaux }\end{array}$} & \multicolumn{2}{|c|}{1} \\
\hline $\begin{array}{l}\text { Période et âge } \\
\text { cles agneaux }\end{array}$ & I.ait & & $\begin{array}{l}\text { Matjère sèche } \\
\text { ingérće }(g / \mathrm{j})\end{array}$ & $\begin{array}{l}\text { Coefficient } \\
\text { d'utilisation } \\
\text { digestive de la } \\
\text { matière sèche }\end{array}$ \\
\hline \multirow[t]{2}{*}{$\cdots \cdots-$} & & -- & $\ldots$ & $\cdots$ \\
\hline & 1 & 8 & $223( \pm 21)$ & $95,0( \pm 1,3)$ \\
\hline 1 re période & 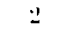 & ii & $\lg (+51)$ & $95,2( \pm 1,6)$ \\
\hline \multirow[t]{2}{*}{2 semaines } & 3 & 5 & $208( \pm 28)$ & $95,1( \pm 1,6)$ \\
\hline & 1 & 8 & $227( \pm 23)$ & $95,9( \pm 1,1)$ \\
\hline & 1 & 5 & $259( \pm 23)$ & $95,5( \pm 1,2)$ \\
\hline 2e pciriode & 2 & 4 & $215( \pm 3(0)$ & $96,2( \pm 1,7)$ \\
\hline \multirow[t]{2}{*}{3 semaines } & ; & 4 & $267( \pm 23)$ & $95,7( \pm 0,8)$ \\
\hline & ' & ii & $238( \pm 55)$ & $95,9( \pm 0,8)$ \\
\hline
\end{tabular}

TABLEAU 2

Agneaux à l'engrais

Digestibilité d'un régime constitué de 85 p. I oo d'aliment concentré et de i 5 p. Ioo de foin de pré

\begin{tabular}{|c|c|c|c|c|c|}
\hline & & & & & \\
\hline $\begin{array}{l}\text { Nombre } \\
\text { d'agneaux }\end{array}$ & Poids vif $(\mathrm{kg})$ & $\begin{array}{l}\text { Quantité fòres } \\
\text { fraîches }(\mathrm{g} / \mathrm{j})\end{array}$ & $\begin{array}{l}\text { Matière sc̀che } \\
\text { ingcrece }(\mathrm{g} / \mathrm{j})\end{array}$ & $\begin{array}{c}\text { Pourcentage } \\
\text { de matière sc̀che } \\
\text { des fèces }\end{array}$ & $\begin{array}{l}\text { Coefficient } \\
\text { d'utilisation } \\
\text { digestive de la } \\
\text { matière sèche }\end{array}$ \\
\hline -... . & & & --1 & -... & $\ldots$ \\
\hline ' & 30 à 35 & $284 \pm 50$ & $82 \cdot( \pm 118)$ & $\{6,9( \pm 2,2)$ & $83,6( \pm 1,3)$ \\
\hline
\end{tabular}

\section{TABLEAU 3}

Brebis en fâturi

Utilisation de la mesure de la quantité de matière sèche excrétée pour estimer, à partir de la digestibilité du fourrage,

la quantité de matière sèche ingérée

\begin{tabular}{|c|c|c|c|c|}
\hline Brebis & $\begin{array}{l}\text { Poids vif } \\
(\mathrm{kg})\end{array}$ & $\begin{array}{l}\text { Quantité de fèces } \\
\text { fraîches }(x / j)\end{array}$ & $\begin{array}{c}\text { Pourcentage } \\
\text { de matière sèche } \\
\text { des fèces }\end{array}$ & $\begin{array}{l}\text { Estimation de } \\
\text { la matière sèrhe } \\
\text { ingérće }(\mathrm{g} / \mathrm{j})\end{array}$ \\
\hline- & - -. & - & & $\cdots-$ \\
\hline 1 & 51 & $2447( \pm 390)$ & $30,1( \pm 3,1)$ & 1781 \\
\hline 2 & 61 & $1860( \pm 514)$ & $35,2( \pm 2,6)$ & 1565 \\
\hline 3 & 52 & $1982( \pm 300)$ & $31,6( \pm 6,9)$ & 1623 \\
\hline
\end{tabular}


de mesurer la digestibilité des aliments dans des conditions plus proches de la normale et d'estimer de façon assez précise les quantités ingérées par des brebis en pâture. Ils nous ont donné entière satisfaction car ils sont bien supportés par les animaux et d'utilisation facile ct rapide.

Reģu pour publication en avril 1975.

\section{REMERCIEMEN'TS}

Nous remercions MM J. FLeChet et E. Diez pour lcur participation à la réalisation de ces équipements.

\section{SUMMARY}

\section{EQUIPMENT FOR TOTAI, COLLECTION OF FAECES FROM IAMBS DURING SUCKLING OR FATTENING AND FOR EWES}

The main particularity of the equipments described is that they are all composed of rigid moulded plastic containers which do not collapse and which keep their initial shape.

For suckling lambs, a beaker of $\mathrm{I} 5 \mathrm{O} \mathrm{ml}$ is put into a strong canvas bag (collection bag) fitted around the anus of the animal (fig. I). The originality of the apparatus is that the harness is replaced by pieces of gunny sticking directly to the fleece.

The bag for fattening lambs made in the same way as that used for suckling lambs, but it is larger and the beaker is replaced by a plastic pail with a capacity of 2.51 (fig. 2). A skin harness is necessary for this equipment (fig. 3).

In the case of the ewes, a 51 plastic can maintained by a harness is used as collection bag (fig. 4 and 5). Perforations of the can allows to eliminate the urine.

\section{RÉFÉRENCES BIBLIOGRAPHIQUES}

ANDREWS R. P., KAI M., ORskov I. R., I969. The effect of different dietary energy concentrations on the voluntary intake and growth of intensively fed lambs. Anim. Prod., 11, I73.

Arnold G. W., I960. Harness for the collection of faeces from grazing ewe and wether sheep. Anim. Prod., 2, I69-173.

Brisson G. J., Bouchard R., Morisset-Rociette M., r97o. Equipment for nutritional studies with lambs weaned at 3 days of age and reared on experimental diats. J. Anim. Sci., 31, 4I7-42I.

Molénat G., Theriez M., r974. L'allaitement artificiel des agneaux. V. Comparaison de différentes concentrations du lait de remplacement. Ann. Zootech., 23, 49I-502.

Royal W. M., 1968. Equipment for collection of faeces from sheep. Proc. Aust. Soc. Anim. Prod., 7, $450-454$.

Theriez M., Protais M., Molknat G., I974. L'allaitement artificiel des agneaux. IV. Compar aison de différentes sources de matières azotées en remplacement de celles du lait. Ann. Zootech., 23, 325-34I. 